\title{
The Prognostic Significance of Different Definitions for Angiosome-Targeted Lower Limb Revascularization
}

\section{Spillerova, Kristyna}

2017-04

Spillerova , K, Biancari , F , Settembre , N , Albäck , A \& Venermo , M 2017 , ' The Prognostic Significance of Different Definitions for Angiosome-Targeted Lower Limb

Revascularization ' , Annals of Vascular Surgery , vol. 40 , pp. 183-189 . https://doi.org/10.1016/j.avsg.2016.06.040

http://hdl.handle.net/10138/236995

https://doi.org/10.1016/j.avsg.2016.06.040

publishedVersion

Downloaded from Helda, University of Helsinki institutional repository.

This is an electronic reprint of the original article.

This reprint may differ from the original in pagination and typographic detail.

Please cite the original version. 


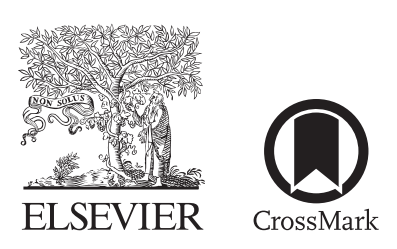

\title{
The Prognostic Significance of Different Definitions for Angiosome-Targeted Lower Limb Revascularization
}

\author{
Kristýna Špillerová, ${ }^{l}$ Fausto Biancari, ${ }^{2}$ Nicla Settembre, ${ }^{l}$ Anders Albäck, ${ }^{1}$ \\ and Maarit Venermo, ${ }^{1}$ Helsinki, and Oulu, Finland
}

\begin{abstract}
Background: The definition of angiosome-targeted revascularization is confusing, especially when a tissue lesion affects several angiosomes. Two different definitions of direct revascularization exist in the literature. The study aim was (1) to compare the 2 definitions of direct revascularization in patients with foot lesions involving more than one angiosome and (2) to evaluate which definition better predicts clinical outcome.

Methods: This study cohort comprises 658 patients with Rutherford 5-6 foot lesions who underwent infrapopliteal endovascular or surgical revascularization between January 2010 and July 2013. We compared the 2 angiosome-targeted definitions using multivariate analysis; the impact of each angiosome-targeted definition was adjusted for a propensity score obtained by means of nonparsimonious logistic regression.

Results: Direct revascularization according to definition A was performed in 367 cases (55.8\%) versus 198 cases $(30.1 \%)$ with definition $B$. The propensity-score-adjusted analysis showed that definition $\mathrm{A}$ of direct revascularization was associated with significantly better wound healing $(P<0.044$, hazard ratio $[\mathrm{HR}] 1.291)$ and lower amputation rates $(P<0.047, \mathrm{HR} 0.706)$, whereas definition B was associated only with significantly better wound healing $(P<0.029$, HR 1.321). The prognostic ability of direct revascularization according to definition $A$ was confirmed in a Cox proportional hazard analysis.

Conclusions: Definition A of direct revascularization was associated with a significantly higher wound healing and leg salvage rate than indirect revascularization in both series. Therefore, it seems that, if the wound spreads over several angiosomes in the forefoot or heel, any angiosome involved in the wound can be targeted.
\end{abstract}

\section{INTRODUCTION}

Recently, the angiosome concept has offered a new perspective on revascularization in patients with critical limb ischemia (CLI) and tissue loss. An

Conflicts of interest: None.

Funding: Finska Läkaresällskapet, Finnish Society for Angiology.

${ }^{I}$ Department of Vascular Surgery, University of Helsinki and Helsinki University Hospital, Helsinki, Finland.

${ }^{2}$ Department of Surgery, Oulu University Hospital, Oulu, Finland.

Correspondence to: Kristýna Špillerová, MD, Department of Vascular Surgery, Helsinki University Hospital, P.O. Box 440, 00029 HUS Helsinki, Finland; E-mail: kspillerova@gmail.com

Ann Vasc Surg 2017; 40: 183-189

http://dx.doi.org/10.1016/j.avsg.2016.06.040

(C) 2016 Elsevier Inc. All rights reserved.

Manuscript received: April 11, 2016; manuscript accepted: June 30 , 2016; published online: 29 September 2016 angiosome was defined by Taylor and Palmer ${ }^{1}$ as a three-dimensional block of tissue supplied and drained by specific, "angiosomal vessels."

Attinger et al. ${ }^{2}$ presented that the foot consist of 6 angiosome regions, each supplied by one of the crural arteries and its terminal branches. The concept of angiosome-targeted (direct) revascularization is based on this division. Direct revascularization (DR) refers to the selective revascularization of the specific artery feeding the angiosome that is affected by an ulcer. If angiosome-targeted revascularization is successful, direct flow from the abdominal aorta to the angiosomal vessel is achieved. ${ }^{3,4}$

Two meta-analyses, including a total of 15 studies, have reported that wound healing and limb salvage, especially in patients with diabetes, are better after DR. ${ }^{5,6}$ However, the definition of 
DR is not clear and, in many of the studies, the definition is lacking altogether. Most of the studies accept an approach as DR if the angiosome with the affected surface is revascularized, with the exception of the forefoot and heel where any angiosome involved in the wound can be targeted. $3,4,7-11$ This exception, however, does not apply to a new definition suggested by Alexandrescu ${ }^{12}$ in his book, Angiosomes Application in Critical Limb Ischemia in Search for Relevance. DR for wounds located in the forefoot and heel is defined as a procedure on the posterior tibial artery only. Furthermore, the definition of the angiosome-guided approach in cases where the wound spans several angiosome regions is scarce.

As recently reported, the wound is located across more than one angiosome in the majority of patients with CLI and tissue lesions. ${ }^{13}$ There is a high predominance of tissue loss located in the toes, metatarsal heads, and the heel. ${ }^{14}$ Therefore, we aimed to investigate which of the 2 existing definitions of DRs predicts better wound healing and leg salvage.

\section{MATERIALS AND METHODS}

The study plan was accepted by the Ethical Committee of the Helsinki University Central Hospital.

\section{Patients}

This retrospective study included 774 consecutive patients with CLI and tissue loss (Rutherford 5-6), who underwent infrapopliteal endovascular or surgical revascularization in our institution between January 2010 and June 2013. Only patients with a foot lesion spreading over several angiosomes or located in more than one angiosome were included. Exclusion criteria were previous infrainguinal revascularization and incomplete information on the status of the foot.

\section{Definitions}

An ischemic foot ulcer was defined as a fullthickness skin defect distal to the malleolar level presented for at least 2 weeks with a toe pressure of $<50 \mathrm{~mm} \mathrm{Hg}$. If toe pressure could not be obtained for some reason (pain, noncompressible artery, or necrosis), the transcutaneous partial pressure of oxygen was measured, with a level of $<30 \mathrm{~mm} \mathrm{Hg}$ indicating an ischemic lesion.

The revascularization techniques in our institution have been described earlier., ${ }^{3,15}$ Our policy is to perform percutaneous transluminal angioplasty (PTA) as a first-line revascularization in stenotic lesions, short occlusions, and in patients who have increased risk for bypass surgery or no autologous vein available. In case of long occlusions in patients who are fit for surgery and do have autologous vein, the policy is to do bypass first. Before PTA, all patients were taking aspirin (100 mg/day). All patients continued life-long aspirin therapy accompanied with clopidogrel for 3 months $(75 \mathrm{mg} /$ day) after the procedure. Patients treated by endovascular approach first had isolated infrapopliteal lesion in 343 (78.5\%) cases; no stent was used for these crural lesions. For bypass surgery, the single-segment great saphenous vein graft (nonreversed, placed under fascia) was used in $64.3 \%$, spliced vein grafts in $30.3 \%$, composite vein with prosthesis in $4.1 \%$, and prosthetic graft in $1.4 \%$. Intraoperative heparin was administered before graft insertion. The patients received lowmolecular-weight heparin ( $1 \mathrm{mg} / \mathrm{kg} /$ day) during their hospital stay accompanied with life-long aspirin therapy ( $100 \mathrm{mg} /$ day) unless contraindicated.

In the literature, 2 definitions of DR in the case of a foot ulcer spreading over the forefoot and heel are available. Definition A accepts DR if any of the affected angiosomes are revascularized ${ }^{3,7-11}$; for example, if the lesion is located in the tip of the toes or the toes are amputated due to gangrene, procedures on either the anterior tibial artery/pedal artery or the posterior tibial artery/plantar arteries, or both, are considered angiosome-targeted. While definition B only accepts the revascularization of posterior tibial artery/plantar arteries as an angiosome-targeted procedure ${ }^{12}$ (Table I).

In cases of a foot ulcer spreading over several angiosomes in other location than the forefoot or heel, we adopted the same approach as described in the study by Iida et al. ${ }^{16,17}$ DR was defined as a procedure on the artery supplying the largest surface of the angiosome involved in the lesion. For example, large necrotic lesion in the dorsum foot of spreading to the medial foot instep, or lesion located at the level of lateral malleolus continuing to dorsum of the foot. In such case, arteria tibialis anterior \pm arteria dorsalis pedis would be the corresponding artery for revascularization.

If the patient suffered from multiple foot ulcers located in separate angiosomes, all affected angiosomes had to be revascularized for the intervention to count as DR.

\section{Wound Location}

We adopted the general scheme of angiosomal distribution $^{3}$ to evaluate the number of affected angiosomes. Ever since we started the research on angiosome concept in 2009, ischemic tissue defect 
Table I. Scheme showing which artery needs to be revascularized in cases where the wound spreads over several angiosomes in the location of the forefoot or heel

\begin{tabular}{|c|c|c|c|c|}
\hline \multirow{2}{*}{$\frac{\text { Revascularization }}{\text { Wound location }}$} & \multicolumn{2}{|l|}{ Definition A } & \multicolumn{2}{|l|}{ Definition B } \\
\hline & Direct & Indirect & Direct & Indirect \\
\hline Forefoot & $\mathrm{ATA} / \mathrm{ADP} \pm \mathrm{ATP} /$ plantar arteries & $\mathrm{AF}$ & $\mathrm{ATP} /$ plantar arteries & ATA, AF \\
\hline Heel & $\mathrm{AF} \pm \mathrm{ATP} /$ plantar arteries & $\mathrm{ATP} / \mathrm{ADP}$ & $\mathrm{ATP} /$ plantar arteries & ATA, AF \\
\hline
\end{tabular}

ADP, arteria dorsalis pedis; AF, arteria fibularis; ATA, arteria tibialis anterior; ATP, arteria tibialis posterior.

and its location have been carefully described in the patient's records. Therefore, the location and severity of the tissue loss was obtained by reviewing our clinical notes by 2 observers, a trainee of vascular surgery with anatomical background and a vascular surgeon with clinical experience more than 15 years. In 95 cases, photographs were available and compared with clinical notes. In many cases, the tissue defect was severe (gangrene, infection) and required periprocedural digit amputation or metatarsal amputation. In such cases, the wound was immediately affecting 2 or 3 angiosomes, depending on the amputation level.

\section{Wound Healing and Follow-up}

The wound care depended on the characteristics of each lesion: debridement of necrotic tissue, surgical revision of infected ulcers together with microbial therapy, and the application of a skin graft in cases where primary or secondary closure was not possible. ${ }^{3}$ A healed wound was defined as complete epithelialization of the tissue defect by secondary intent or after any additional local ulcer surgery. The wound was considered nonhealed if it was still open at the end of the follow-up.

After revascularization, patients remained under routine surveillance in the outpatient clinic by a vascular nurse who carried out a duplex ultrasound examination of the revascularized artery and followed the foot status; a vascular surgeon was consulted if necessary. In the case of PTA, the visits were scheduled at 1,3 , and 6 months after revascularization, and in the case of a bypass graft at 1, 3, 6, and 12 months after revascularization. If the duplex examination of bypass graft showed signs of stenosis, the patient underwent control digital subtraction angiogram (DSA) within 2 weeks; a PTA to bypass graft was performed in case of confirmed stenosis. If the wound opened after routine duplex surveillance ended, the patient continued visits to the outpatient clinic until the wound healed. The follow-up ended and the wound was considered as nonhealed if the patient underwent a new infrainguinal bypass due to failure of primary intervention (endovascular or surgical), new additional PTA of crural arteries after primary intervention, or a major amputation due to nonhealing foot ulcer or occluded bypass graft, or if the patient died.

\section{Data Collection}

Data collection was performed using our prospectively collected database and scrutinizing it retrospectively by reviewing patient records as well as the patient's imaging files. In patients who underwent endovascular treatment, DSAs before and after revascularization were reviewed to evaluate whether an angiosome-targeted procedure had been performed, and in patients undergoing surgical bypass, the preoperative magnetic resonance angiograms and DSAs, if available, were reviewed as well. All patients treated by PTA had on-table DSA right after revascularization as a control of possible complications (dissection, microembolism).

The patient's baseline characteristics and operative data are summarized in Table II.

Estimated glomerular filtration rate (eGFR) was estimated by the Modification of Diet in Renal Disease formula (Levey).

\section{Outcome Measures}

We compared the outcome of DR versus indirect revascularization (IR) using the 2 above-described definitions. The primary outcome measures were wound healing and leg salvage. Survival was a secondary outcome end point.

\section{Statistical Analysis}

Statistical analysis was performed using SPSS version 22.0 statistical software (IBM SPSS, Inc., Chicago, IL). No attempt to replace missing values was made. Fisher's exact test, the chi-squared test, and the Mann-Whitney and Kaplan-Meier tests were used for univariable analysis. Multivariate analysis for assessing the impact of baseline variables on late outcome was performed using the Cox proportional hazards method. The impact of each DR definition was adjusted for a propensity 
Table II. Baseline characteristics of patients undergoing lower limb revascularization according to 2 different definitions of direct (angiosome-targeted) revascularization

\begin{tabular}{|c|c|c|c|c|c|c|}
\hline \multirow[b]{2}{*}{ Variable } & \multicolumn{3}{|l|}{ Definition A } & \multicolumn{3}{|l|}{ Definition B } \\
\hline & $\begin{array}{l}\text { Indirect } 291 \\
\text { patients }\end{array}$ & $\begin{array}{l}\text { Direct } 367 \\
\text { patients }\end{array}$ & $P$ value & $\begin{array}{l}\text { Indirect } 460 \\
\text { patients }\end{array}$ & $\begin{array}{l}\text { Direct } 198 \\
\text { patients }\end{array}$ & $P$ value \\
\hline Age & $75.5 \pm 11.3$ & $73.8 \pm 11.1$ & 0.047 & $75.6 \pm 11.0$ & $72.1 \pm 11.5$ & $<0.0001$ \\
\hline Female & $107(36.8)$ & $139(37.9)$ & 0.771 & $181(39.3)$ & $65(32.8)$ & 0.113 \\
\hline Smoking habit & $41(14.1)$ & $55(15.0)$ & 0.746 & $61(13.3)$ & $35(17.7)$ & 0.141 \\
\hline Pulmonary disease & $38(13.1)$ & $37(10.1)$ & 0.233 & $58(12.6)$ & $17(8.6)$ & 0.136 \\
\hline Atrial fibrillation & $94(32.3)$ & $99(27.0)$ & 0.136 & $147(32.0)$ & $46(23.2)$ & 0.024 \\
\hline Hypertension & $195(67.0)$ & $220(59.9)$ & 0.062 & $293(63.7)$ & $122(61.6)$ & 0.612 \\
\hline Estimated glomerular filtration rate & $74.9 \pm 36.1$ & $76.3 \pm 39.2$ & 0.663 & $74.8 \pm 36.4$ & $77.9 \pm 41.0$ & 0.360 \\
\hline Dialysis & $11(3.8)$ & $29(7.9)$ & 0.028 & $22(4.8)$ & $18(9.2)$ & 0.048 \\
\hline Kidney transplantation & $4(1.4)$ & $6(1.6)$ & 0.786 & $4(0.9)$ & $6(3.0)$ & 0.074 \\
\hline Diabetes & $176(60.5)$ & $233(63.5)$ & 0.430 & $280(60.9)$ & $129(65.2)$ & 0.299 \\
\hline Coronary artery disease & $118(40.5)$ & $127(34.6)$ & 0.117 & $180(39.1)$ & $65(32.8)$ & 0.125 \\
\hline Heart failure & $39(13.4)$ & $45(12.3)$ & 0.663 & $60(13.0)$ & $24(12.1)$ & 0.745 \\
\hline Stroke & $44(15.1)$ & $48(13.1)$ & 0.453 & $65(14.1)$ & $27(13.6)$ & 0.867 \\
\hline C-reactive protein (mg/dL) & $46.3 \pm 53.8$ & $47.9 \pm 54.2$ & 0.835 & $49.5 \pm 54.4$ & $41.7 \pm 52.5$ & 0.004 \\
\hline C-reactive protein $>10 \mathrm{mg} / \mathrm{dL}$ & $197(67.7)$ & $254(69.2)$ & 0.678 & $330(71.7)$ & $121(61.1)$ & 0.007 \\
\hline Gangrene & $69(23.7)$ & $124(33.8)$ & 0.005 & $138(30.0)$ & $55(27.8)$ & 0.566 \\
\hline No. of affected angiosomes & $2.3 \pm 1.1$ & $2.2 \pm 0.7$ & 0.815 & $2.3 \pm 0.9$ & $2.0 \pm 0.8$ & $<0.0001$ \\
\hline Complete pedal arch & $45(15.5)$ & $87(23.7)$ & 0.009 & $79(17.2)$ & $53(26.8)$ & 0.005 \\
\hline Bypass surgery & $88(30.2)$ & $133(36.2)$ & 0.106 & $143(31.1)$ & $78(39.4)$ & 0.039 \\
\hline
\end{tabular}

score obtained by nonparsimonious logistic regression including all variables listed in Table I. Separate propensity scores were estimated for each definition. The regression models were calibrated by the Hosmer-Lemeshow Goodness-of-Fit Test. Model discrimination was evaluated using the area under the receiver operating characteristic (ROC) curve. All tests were two-sided with the alpha level set at 0.05 for statistical significance.

\section{RESULTS}

After the exclusion criteria, the cohort resulted in a total of 658 patients, of which $437(66.4 \%)$ were treated by PTA and 221 (33.6\%) by infrapopliteal surgical bypass. Of 658 patients, $367(55.8 \%)$ fulfilled the criteria for direct lower limb revascularization when definition A was used. When definition B was applied, the number of cases who underwent DR was significantly smaller, 198 (30.1\%, $P<0.05)$. The baseline and operative characteristics in patients with DR compared with those with IR according to both definitions are summarized in Table II. The mean follow-up was 21 months (standard deviation 13.8 months, range $0-51$ ). In the endovascular group, $29.9 \%$ of patients underwent reintervention compared with $16.7 \%$ in bypass surgery (Fig. 1). Two hundred three (30.9\%) patients

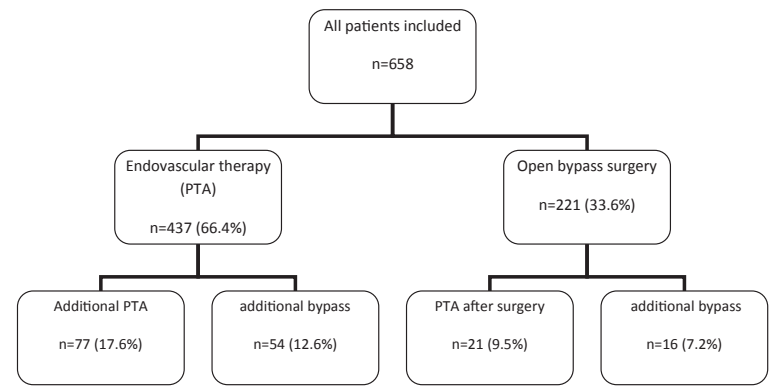

Fig. 1. Reintervention rates according to revascularization method.

underwent minor amputation (130 toe and 73 metatarsal amputations) during the follow-up. It is worth noting that DR according to definition A resulted in a rather balanced distribution of baseline characteristics. When examining definition A of DR, the patients were significantly younger and a higher proportion of them had a complete pedal arch visible at angiography, but they also had a significantly higher prevalence of dialysis and foot gangrene than patients who underwent IR (Table II). In contrast, patients who underwent DR according to definition $\mathrm{B}$ were also significantly younger and had a lower prevalence of atrial fibrillation, a lower number of affected angiosomes, a higher prevalence of a complete pedal arch at angiography, 
Table III. Propensity-score-adjusted outcome according to 2 different definitions of direct revascularization

\begin{tabular}{llllll}
\hline & \multicolumn{2}{l}{ Definition A } & & \multicolumn{2}{l}{ Definition B } \\
\cline { 2 - 3 } \cline { 5 - 6 } Outcome end points & $P$ value & HR $(95 \%$ CI $)$ & & $P$ value & HR (95\% CI) \\
\hline Wound healing & 0.044 & $1.291(1.007-1.656)$ & & 0.029 & $1.321(1.029-1.695)$ \\
Major amputation & 0.047 & $0.706(0.501-0.996)$ & & 0.096 & $0.697(0.456-1.066)$ \\
Mortality & 0.938 & $0.990(0.761-1.286)$ & & 0.087 & $0.764(0.561-1.040)$ \\
\hline
\end{tabular}

Table IV. Outcome end points adjusted for diabetes, estimated glomerular filtration rate, C-reactive protein, revascularization method, number of affected angiosomes, and presence of intact pedal arch according to 2 different definitions of direct revascularization

\begin{tabular}{|c|c|c|c|c|}
\hline \multirow[b]{2}{*}{ Outcome end points } & \multicolumn{2}{|c|}{ Definition A } & \multicolumn{2}{|c|}{ Definition B } \\
\hline & $P$ value & $\operatorname{HR}(95 \% \mathrm{CI})$ & $P$ value & HR $(95 \%$ CI $)$ \\
\hline Wound healing & 0.037 & $1.294(1.016-1.648)$ & 0.060 & $1.267(0.990-1.621)$ \\
\hline Major amputation & 0.044 & $0.703(0.847-0.990)$ & 0.045 & $0.652(0.429-0.990)$ \\
\hline Mortality & 0.356 & $0.886(0.685-1.146)$ & 0.043 & $0.733(0.543-0.991)$ \\
\hline
\end{tabular}

and lower baseline levels of C-reactive protein; however, they had a higher prevalence of dialysis than their counterparts who underwent IR.

Unadjusted actuarial analysis showed that, irrespective of the definition (A or B) that was used, DR yielded better wound healing and leg salvage rates when compared with IR: if definition A was applied, the wound healing rates in the DR versus IR groups at 1 year were $72.3 \%$ vs. $66.6 \%$ (logrank: $P=0.031)$, respectively, and the leg salvage rates at 1 year were $83.4 \%$ vs. $75.6 \%(P=0.019)$, respectively. If definition $B$ was applied, the respective wound healing rates at 1 year were $74.9 \%$ vs. $67.3 \%$ (log-rank: $P=0.019$ ) and the respective leg salvage rates at 1 year were $87.4 \%$ vs. $76.8 \%$ $(P=0.003)$.

A logistic regression model including all variables listed in Table II provided a propensity score for definition A of DR (Hosmer-Lemeshow test: $P=0.183$ ) with an area under the ROC curve of 0.635 (95\% confidence interval [CI] 0.593-0.677) and for definition B a propensity score (Hosmer-Lemeshow test: $P=0.659)$ with an area under the ROC curve of 0.686 (95\% CI $0.643-0.729)$.

A propensity-score-adjusted analysis showed that, when definition A was adopted, DR was associated with significantly better wound healing and leg salvage rates, whereas when definition $\mathrm{B}$ was applied, DR was associated only with significantly better wound healing (Table III).

The prognostic ability of definition A was confirmed in a Cox proportional hazard analysis as adjusted for diabetes, eGFR, C-reactive protein, revascularization method, number of affected angiosomes, and the presence of an intact pedal arch (Table IV). This model also showed a significant predictive value of DR according to definition B for leg salvage and mortality, but only a trend toward better wound healing. When both DR definitions were included in the latter regression model, only definition A was associated with better wound healing $(P=0.040$, hazard ratio [HR] $1.286,95 \%$ CI $1.012-1.635)$ and a lower risk of major amputation $(P=0.038$, HR $0.698,95 \%$ CI 0.497-0.980).

\section{DISCUSSION}

Even though 2 recent meta-analyses ${ }^{4,5}$ show a tendency toward better clinical outcome after angiosome-guided revascularization, many clinicians argue against the concept. In our recent study, we demonstrated that, in only $24 \%$ of the patients with CLI and tissue loss, the wound is limited to one angiosome and that, in the majority of cases, 2 or more angiosomes are affected. ${ }^{13}$ Furthermore, we have shown that the number of affected angiosomes is associated with wound healing time and inversely associated with wound healing rate. ${ }^{6}$

The majority of the studies does not report the number of affected angiosomes nor define the DR in cases where the wound affects several angiosomes. ${ }^{3,8-11,18-20}$ The study by Iida et al. ${ }^{16,17}$ targeted 
the largest surface affected; due to the dual blood supply of the heel and digits, however, it is difficult to correctly define the DR of this regions.

In this study, we included an extensive series of patients who had lesions affecting more than one angiosome and in whom 2 different definitions of angiosome-targeted revascularization, as described in the methodology, were tested.

Although both definitions were associated with better wound healing, definition A also seemed to predict leg salvage when the risk factors were adjusted with propensity score analysis. Furthermore, the use of definition B was less successful clinically as it resulted in fewer cases in which DR was performed, $30 \%$ vs. $56 \%$ when definition A was used. This significant difference may be explained by the poorer feasibility of an angiosome-targeted procedure using definition $\mathrm{B}$, as the clinician is limited to the posterior tibial artery as the only option for DR in cases where the wound is located in the forefoot or heel. Furthermore, we experience that the arteria tibialis posterior was in many cases severely diseased with long occlusions or multiple (pearl-like) lesions, therefore the easier artery was chosen for endovascular treatment.

It is worth noting that the patients in the targeted revascularization group were divided in terms of their characteristics when definition A was compared with definition B. Patients for whom DR was achieved according to definition B had fewer comorbidities and less severe foot lesions as graded by the number of affected angiosomes, in addition to having lower baseline levels of C-reactive protein when compared with the patients treated with DR according to definition A.

While the study by Higashimori et al. ${ }^{21}$ demonstrates that an existing intact pedal arch is essential when only one vessel runoff can be established to the foot, another study by Rashid et al. ${ }^{22}$ also suggests that the quality of the pedal arch is more important with regard to wound healing time than whether or not angiosome-guided revascularization is achieved. In this study, we evaluated the intactness of the pedal arch and found that patients with DR more often had an open pedal arch. However, this factor was adjusted with propensity score analysis and should not have an impact on the results.

To confirm the finding of the propensity score analysis, a Cox proportional hazards model was utilized. This multivariate analysis confirmed the finding with regard to definition $\mathrm{A}$. In the Cox model, definition B also predicted leg salvage, but yielded only a trend toward better wound healing. The Cox model supports the superiority of definition A with regard to the angiosome concept.
Based on our findings, it seems that, if the wound spreads over more than one angiosome in the location of the forefoot or heel, any angiosomal artery involved in the wound can be targeted to achieve a better clinical outcome. Therefore, the clinician has more options to choose from, with a higher probability that revascularization will be possible in one of the arteries.

This is a retrospective study, and its main limitation is the description of wound location, which is extracted from case records. Even though wound location has been carefully reported in our institution ever since the emergence of interest in angiosomes and wound location in the literature, determination of the affected angiosome can be difficult in some cases. Furthermore, the study by Varela et al. $^{4}$ showed the importance of collaterals in wound healing; they concluded that IR via collateral with good diameter can provide similar result as DR. This cohort, however, does not provide the information of collateralization and therefore it is a limitation to our study as it could influence our results.

Furthermore, in cases where graft occluded shortly after bypass surgery $(n=15)$ the wound was considered nonhealed in follow-up as reprocedure or major amputation was performed. Unsuccessful wound healing, however, was related to surgical failure rather than angiosome-guided revascularization.

The research on angiosome concept lacks a well-planned prospective study in which the wound location and size are defined precisely and where high-quality angiograms with information on collaterals as well as the patency of the pedal arch are performed and the wound healing is followed carefully. This kind of study would yield definitive information on the true influence of angiosome-targeted revascularization on leg salvage. The evidence so far, although based almost solely on retrospective reports and therefore not definitive, should guide interventionists toward the direction of angiosometargeted revascularization when CLI patients with tissue lesions are treated.

\section{CONCLUSION}

Consensus needs to be achieved regarding the accurate definition of DR, especially if more than one angiosome is clinically involved. In this series, the proportion of patients meeting the criteria of DR using definition A was larger than when definition B was used. Definition A of DR was associated with a significantly higher wound healing and leg salvage rate than IR, and its prognostic significance was 
not inferior to definition B. Based on our findings, it seems that, if the wound spreads over more than one angiosome in the location of the forefoot or heel, any angiosome involved in the wound can be targeted to achieve a better clinical outcome.

\section{REFERENCES}

1. Taylor GI, Palmer JH. The vascular territories (angiosomes) of the body: experimental study and clinical applications. Br J Plast Surg 1987;40:113-41.

2. Attinger CE, Evans KK, Bulan E, et al. Angiosomes of the foot and ankle and clinical implications for limb salvage: reconstruction, incisions, and revascularization. Plast Reconstr Surg 2006;117:261S-93S.

3. Söderström M, Albäck A, Biancari F, et al. Angiosome-targeted infrapopliteal endovascular revascularization for treatment of diabetic foot ulcers. J Vasc Surg 2013;57:427-35.

4. Varela C, Acín F, de Haro J, et al. The role of foot collateral vessels on ulcer healing and limb salvage after successful endovascular and surgical distal procedures according to an angiosome model. Vasc Endovascular Surg 2010;44:654-60.

5. Bosanquet DC, Glasbey JC, Williams IM, et al. Systematic review and meta-analysis of direct versus indirect angiosomal revascularisation of infrapopliteal arteries. Eur J Vasc Endovasc Surg 2014;48:88-97.

6. Biancari F, Juvonen T. Angiosome-targeted lower limb revascularization for ischemic foot wounds: systematic review and meta-analysis. Eur J Vasc Endovasc Surg 2014;47:517-22.

7. Spillerova K, Biancari F, Leppäniemi A, et al. Differential impact of bypass surgery and angioplasty on angiosometargeted infrapopliteal revascularization. Eur J Vasc Endovasc Surg 2015;49:412-9.

8. Azuma N, Uchida H, Kokubo $\mathrm{T}$, et al. Factors influencing wound healing of critical ischaemic foot after bypass surgery: is the angiosome important in selecting bypass target artery? Eur J Vasc Endovasc Surg 2012;43:322-8.

9. Kret MR, Cheng D, Azarbal AF, et al. Utility of direct angiosome revascularization and runoff scores in predicting outcomes in patients undergoing revascularization for critical limb ischemia. J Vasc Surg 2014;59:121-8.

10. Acín F, Varela C, López de Maturana I, et al. Results of infrapopliteal endovascular procedures performed in diabetic patients with critical limb ischemia and tissue loss from the perspective of an angiosome-oriented revascularization strategy. Int J Vasc Med 2014;2014: 270539.

11. Neville RF, Attinger CE, Bulan EJ, et al. Revascularization of a specific angiosome for limb salvage: does the target artery matter? Ann Vasc Surg 2009;23:367-73.

12. Alexandrescu V. Angiosomes application in critical limb ischemia in search for relevance. Turin, Edizioni Minerva Medica S.p.A 2013:1-11

13. Špillerová K, Sörderström M, Albäck A, et al. The feasibility of angiosome-targeted endovascular treatment in patients with critical limb ischaemia and foot ulcer. Ann Vasc Surg 2016;30:270-6.

14. Aerden D, Denecker N, Gallala S, et al. Wound morphology and topography in the diabetic foot: hurdles in implementing angiosome-guided revascularization. Int J Vasc Med 2014;2014:672897.

15. Söderström M, Arvela EM, Korhonen M, et al. Infrapopliteal percutaneous transluminal angioplasty versus bypass surgery as first-line strategies in critical leg ischemia: a propensity score analysis. Ann Surg 2010;252:765-73.

16. Iida O, Takahara M, Soga $Y$, et al. Impact of angiosomeoriented revascularization on clinical outcomes in critical limb ischemia patients without concurrent wound infection and diabetes. J Endovasc Ther 2014;21:607-15.

17. Iida O, Takahara M, Soga Y, et al. Worse limb prognosis for indirect versus direct endovascular revascularization only in patients with critical limb ischemia complicated with wound infection and diabetes mellitus. Eur J Vasc Endovasc Surg 2013;46:575-82.

18. Osawa S, Terashi H, Tsuji Y, et al. Importance of the six angiosomes concept through arterial-arterial connections in CLI. Int Angiol 2013;32:375-85.

19. Oshima S, Noda K, Sumida H, et al. Impact of the angiosome concept for endovascular therapy in patients with critical limb ischemia due to isolated below-the knee lesions. Eur Heart J 2012;33:523.

20. Neville RF, Sidawy AN. Surgical bypass: when is it best and do angiosomes play a role? Semin Vasc Surg 2012;25: $102-71$.

21. Higashimori A, Iida O, Yamauchi Y, et al. Outcomes of One straight-line flow with and without pedal arch in patients with critical limb ischemia. Catheter Cardiovasc Interv 2016;87:129-33.

22. Rashid H, Slim H, Zayed H, et al. The impact of arterial pedal arch quality and angiosome revascularization on foot tissue loss healing and infrapopliteal bypass outcome. J Vasc Surg 2013;57:1219-26. 